\title{
Measuring capability of a Poisson process: Relative goodness of the estimates obtained by different approaches
}

\author{
Surajit Pal ${ }^{1}$, Susanta Kumar Gauri ${ }^{2 *}$ \\ ${ }^{1}$ SQC \& OR Unit, Indian Statistical Institute, 110, N. Manickam Road, Chennai-600029, INDIA \\ ${ }^{2}$ SQC \& OR Unit, Indian Statistical Institute, 203, B. T. Road, Kolkata-700108, INDIA \\ ${ }^{*}$ Corresponding Author: e-mail: susantagauri@hotmail.com, Tel. No.: 091-033-2575-3350; Fax No.: 091-033-2577-6042 \\ ORCID iDs: https://orcid.org/ 0000-0001-6066-5782 (Pal), https://orcid.org/ 0000-0003-4654-7696 (Gauri)
}

\begin{abstract}
In real life, often number of occurrences of some events (e.g. defects/nonconformities, errors, number of customers served etc.) in an inspection unit is considered as an important quality characteristic. Such characteristics are usually smaller-the-better (STB) or larger-the-better (LTB) type and often are modeled by Poisson distribution with parameter $\lambda(>0)$, where $\lambda$ is the rate of occurrence of the event. The capability index $\left(C_{p u}\right.$ or $\left.C_{p l}\right)$ of a Poisson process cannot be estimated by using the standard formulas that are developed for normal processes. Usually some approximate approaches or generalized approaches are used for estimating $C_{p u}$ or $C_{p l}$ of a Poisson process. However, how well these estimates represent the true capability of the process is unknown. Consequently, an index user may inadvertently be led to erroneous decision making. This paper identifies the most appropriate measure of capability of a Poisson process and then assesses relative goodness of the estimates of $C_{p u}$ (or $C_{p l}$ ) obtained by different approaches. It is found that only the estimated $\hat{C}_{p u}$ (or $\hat{C}_{p l}$ ) value obtained by Mapping based approach represents the true capability of a Poisson process. Normal approximation approach and Transformation approach are also quite acceptable for measuring capability of a Poisson process.
\end{abstract}

Keywords: Process capability index, $C_{p u}, C_{p l}$, Poisson process, goodness of estimates of $C_{p u}$ or $C_{p l}$

DOI: http://dx.doi.org/10.4314/ijest.v12i4.1

Cite this article as:

Pal S., Gauri S.K. 2020. Measuring capability of a Poisson process: Relative goodness of the estimates obtained by different approaches. International Journal of Engineering, Science and Technology, Vol. 12, No. 4, pp. 1-13. doi: 10.4314/ijest.v12i4.1

Received: June 13, 2020; Accepted: September 28, 2020; Final acceptance in revised form: October 2, 2020

\section{Introduction}

Process capability refers to the ability of a given process to produce outputs according to specified requirements. The basic process capability index is $C_{p}=(U S L-L S L) / 6 \sigma$, where $U S L$ and $L S L$ are the upper and lower specification limits and $\sigma$ is the population standard deviation. When there is only $U S L$ or only $L S L$ for a product characteristic, then the process capability indices are defined as $C_{p u}=(U S L-\mu) / 3 \sigma$ and $C_{p l}=(\mu-L S L) / 3 \sigma$ respectively, where $\mu$ is the population mean. The most commonly used other indices are $C_{p k}, C_{p m}$ and $C_{p m k}$. All these indices are developed for a product characteristic that can be described as a continuous variable and follows normal distribution. The details about these indices are available in Kane (1986), Kotz and Johnson (1993), English and Taylor (1993), Kotz and Johnson (2002), Vannman (2006), Chen et al. (2008), Wu et al. (2009), Yum and Kim (2011), Grau (2012) and Chen et al. (2017). The generalization of these indices for continuous non-normal variables are suggested by Clements (1989), Pearn and Kotz (1994), Pearn and Chen (1995), Shore (1998), Chen (2000), Goswami and Dutta 
(2013), Wang et al. (2016), Shi et al, (2016), Polhemus (2018) and Chen et al. (2019). A good bibliometric analysis for process capability research is available in Ahmad et al. (2019).

However, there are many practical situations in which number of occurrences of some events (e.g. defects/nonconformities, errors etc.) in an inspection unit is an important consideration for judging the quality of the manufactured products. For example, number of defects per 100 square metre cloth, number of defects per wafer, number of defects per 5 printed circuit board etc. are important quality characteristics for cloth, wafer and printed circuit board respectively. It may be noted that inspection unit is simply an entity for which it is convenient to keep record. It can be a single unit of product or a group of $n$ units of products $(n>$ 1). If the number of opportunities or potential locations for the event be infinitely large and the probability of occurrence of an event at any location be small and constant, then the occurrences of the event in an inspection unit is well modelled by Poisson distribution with parameter $\lambda(>0)$, where $\lambda$ is the rate of occurrence of the event. In fact, any random phenomenon that occurs on a per unit (or per unit area, per unit volume, per unit time, etc.) basis is often well approximated by Poisson distribution (Montgomery, 2009). It may further be noted that a Poisson variable in manufacturing environment is usually smaller-the-better (STB) type variable and it is desired that its values do not exceed USL. The capability of such a process can be judged through measuring $C_{p u}$ value. On the other hand, the Poisson variables in case of service processes are usually larger-the-better (LTB) type, e.g. number of customers served per hour. It is desired that the values of such a variable do not be less than $L S L$, and thus, the capability of a service process can be assessed by measuring $C_{p l}$ value.

It is well known that both the mean and variance of a Poisson random variable $C$ are equal to the parameter $\lambda$, and the distribution is skewed. However, if $\lambda>5$ then $Z=(C-\lambda) / \sqrt{\lambda}$ approximately follows standard normal distribution (Montgomery and Runger, 2014). Therefore, the standard formula can be used for obtaining an approximate estimate of $C_{p u}$ (or $\left.C_{p l}\right)$ from a Poisson process if its parameter $\lambda>5$. Alternatively, percentile based approach (Clement, 1989) may also be used for obtaining an estimate of $C_{p u}$ (or $C_{p l}$ ) from a Poisson process. However, the estimate will be approximate only because the values of percentile points that are to be used in these computations have to be approximated. Thus, both the approaches can measure $C_{p u}$ or $C_{p l}$ of a Poisson process approximately only. Maravelakis (2016) have developed a method for measuring process capability of a Poisson process. In this method, Poisson data are first converted into normally distributed data by using a two-step transformation technique and then, capability of the Poisson process is assessed by directly applying the standard formula for $C_{p u}$ or $C_{p l}$ on the transformed data.

Borges and Ho (2001) suggested a different measure of process capability, called $C$-index, which has one-to-one correspondence (mapping) between the proportion of nonconforming items and Z-value of the standard normal distribution. This implies that the process capability will respond to changes in the nonconforming region and not to changes in the distribution of the observed quality characteristic. Thus, process capability index for Poisson process can be expressed in terms of $C$-index. In case of unilateral specification, the $C_{p u}$ or $C_{p l}$ of a Poisson process can be measured in terms of $C_{u}$ or $C_{l}$ respectively.

In the recent past, researchers have proposed some generalized indices for assessment of process capability, which can be used as alternative to $C_{p}$. These indices are defined as the ratio of two probabilities instead of ratio of the specification width and actual process width. Thus, these indices can be computed irrespective of distribution of the quality characteristics (normal or nonnormal) and data type (continuous or discrete). Yeh and Bhattacharya (1998) proposed $C_{f}$ index, Perakis and Xekalaki (2002, 2005) presented $C_{p c}$ index and Maiti et al. (2010) suggested $C_{p y}$ index for assessment of process capability. In case of unilateral specification, the equivalent indices for $C_{p u}$ can be obtained as $C_{f u}, C_{p c u}$ and $C_{p y u}$, and the equivalent indices for $C_{p l}$ can be obtained as $C_{f l}, C_{p c l}$ and $C_{p y l}$. Therefore, $C_{p u}$ or $C_{p l}$ for a Poisson process can be measured in terms of these generalized indices.

Some of the major uses of the estimates of process capability indices in a manufacturing set up are i) predicting how well the process will hold the specifications, ii) selecting between competing vendors, and iii) assisting product developers/designers in selecting or modifying a process. The estimates of $C_{p u}$ or $C_{p l}$ can be obtained from a Poisson process by using any of the above mentioned approaches. However, the estimates of $C_{p u}$ (or $C_{p l}$ ) obtained from the same process using different approaches usually differ widely. Again, the same value of the estimates of $C_{p u}$ (or $C_{p l}$ ) obtained by different approaches signifies different capabilities for a Poisson process. All these pose difficulties in interpreting the estimates of $C_{p u}$ (or $C_{p l}$ ) obtained by different approaches. Consequently, the index users may unknowingly be led to erroneous decision making. The aim of this study is to assess the relative goodness of the estimates of $C_{p u}$ or $C_{p l}$ obtained by different approaches, so that all the stakeholders of the index can choose the best method for estimating $C_{p u}$ or $C_{p l}$ of a Poisson process and can avoid the methods that are likely to give misleading assessment of process capability.

The article is organized as follows: Different approaches for computation of $C_{p u}$ or $C_{p l}$ from Poisson data are described in Section 2. A procedure to compare the relative goodness of the estimated $\hat{C}_{p u}$ (or $\hat{C}_{p l}$ ) values obtained by various methods is discussed in Section 3. Analysis of multiple case study data sets and related results are presented in Section 4. Important findings and the issues related to different methods are discussed in Section 5. Section 6 concludes the paper. 


\section{Different approaches for computation of $C_{p u}$ or $C_{p l}$ from Poisson data}

For convenience, let us consider that a single unit of product represents an inspection unit. Suppose the number of opportunities for occurrences of an event in an inspection unit is infinitely large and the probability of occurrence of the event is small and constant. Then, if the random variable $C$ denotes the number of occurrences of the event in a unit, then $C$ has a Poisson distribution with parameter $\lambda(>0)$, i.e.

$$
P\{C=c\}=\frac{e^{-\lambda} \lambda^{c}}{c !} ; c=0,1,2, \ldots
$$

Suppose numbers of occurrences of the events are observed in each of the $m$ units collected from a stable process, and $c_{i}$ is the number of events occurred in the $i^{t h}$ unit $(i=1,2,3 \ldots, m)$. Then, the unknown parameter $\lambda$ can be estimated as

$$
\hat{\lambda}=\bar{c}=\sum_{i=1}^{m} c_{i} / m
$$

If the concerned event is STB type, it will have only $U S L$, and one will need to estimate $C_{p u}$. On the other hand, if the concerned event is LTB type, it will have only $L S L$, and one will need to estimate $C_{p l}$. The various approaches that can be used for estimating $C_{p u}$ or $C_{p l}$ from a Poisson process are described in the following sub-sections.

It may be noted that value of $m$ should be sufficiently large and the samples should be collected from an in control process over long period of time. More will be the sample size, better will be the estimate of the population parameter, which in turn gives more accurate estimate of expected proportion of nonconforming units with respect to USL (or LSL) and thus, result in more accurate estimates of process capability indices.

\subsection{Normal approximation approach}

If the estimate of population parameter $\hat{\lambda}(=\bar{c})$ is greater than 5 , the distribution of $c$ may be approximated by normal distribution with mean $\bar{c}$ and variance $\bar{c}$ (Montgomery and Runger, 2014). Thus, the values of $C_{p u}$ and $C_{p l}$ can be approximated as follows:

$$
\begin{aligned}
\hat{C}_{p u} & =\left(c_{U}-\bar{c}\right) /(3 \times \sqrt{\bar{c}}) \\
\hat{C}_{p l} & =\left(\bar{c}-c_{L}\right) /(3 \times \sqrt{\bar{c}})
\end{aligned}
$$

where $c_{U}$ is the $U S L$ for occurrences of a STB type event and $c_{L}$ is the $L S L$ for occurrences of a LTB type event in an inspection unit.

\subsection{Percentile based approach}

Clements (1989) proposed the percetile based approach which is essentially generalization of the proces capability indices for non-normal continuous processes. In this approach, $C_{p u}$ and $C_{p l}$ are estimated as follows:

$$
\begin{aligned}
\hat{C}_{p u} & =\left(c_{U}-M\right) /\left(C_{0.99865}-M\right) \\
\hat{C}_{p l} & =\left(M-c_{L}\right) /\left(M-C_{0.00135}\right)
\end{aligned}
$$

where $M$ is the median ( $50^{\text {th }}$ percentile point), $C_{0.99865}$ is the $99.865^{\text {th }}$ percentile point and $C_{0.00135}$ is the $0.135^{t h}$ percentile point of the non-normal continuous distribution. The same method can be applied to Poisson process for obtaining an approximate estimate of $C_{p u}$ and $C_{p l}$. In case of Poisson distribution, $M, C_{0.99865}$ and $C_{0.00135}$ must be integers and so it may not be possible to get exact $50^{\text {th }}, 99.865^{\text {th }}$ or $0.135^{\text {th }}$ percentile points.

\subsection{Transformation approach}

Maravelakis (2016) proposed a transformation technique by which observed count data $\left(c_{i}\right)$ from Poisson distribution can be transformed into $Q_{i}(i=1,2,3, \ldots, m)$ values. Using the same transformation technique, $c_{U}$ (or $\left.c_{L}\right)$ can also be transformed into $Q$ value. Suppose the transformed $c_{U}$ (or $c_{L}$ ) is denoted as $Q_{U}\left(\right.$ or $\left.Q_{L}\right)$. Quesenberry (1991) has shown that if the Poisson parameter $\lambda$ be constant, then $Q_{i}(i=1,2,3, \ldots, m)$ are approximately independently and normally distributed. Therefore, $C_{p u}$ or $C_{p l}$ of the original process can be evaluated from the $Q_{i}(i=1,2,3, \ldots, m)$ values as follows:

$$
\begin{aligned}
& \hat{C}_{p u}=\left(Q_{U}-\bar{Q}\right) /\left(3 \times S D_{Q}\right) \\
& \hat{C}_{p l}=\left(\bar{Q}-Q_{L}\right) /\left(3 \times S D_{Q}\right)
\end{aligned}
$$


where $\bar{Q}$ and $S D_{Q}$ are average and standard deviation of the $Q_{i}(i=1,2,3, \ldots, m)$ values. Maravelakis (2016) has proposed two different techniques for transformation of count data into $Q$ values for the following two cases.

Case 1: Transformation of Poisson data when $\lambda$ is known

Let defects rate $\lambda=\lambda_{0}$ (known), and $c_{i}$ denotes the number of defects observed in an inspection unit. At first, the sample observations $\left(c_{i}\right)$ are transformed into cumulative Poisson value $\left(u_{i}\right)$ using the Poisson cumulative distribution function, as shown in Equation (9). Then, the cumulative Poisson values are retransformed into $Q_{i}$ values by the inverse of the standard normal distribution as shown in Equation (10).

$$
\begin{aligned}
& u_{i}=F_{P}\left(c_{i} ; \lambda_{0}\right) ; i=1,2,3, \ldots, m \\
& Q_{i}=\Phi^{-1}\left(u_{i}\right) ; i=1,2, \ldots, m
\end{aligned}
$$

Case 2: Transformation of Poisson data when $\lambda$ is unknown

At first, the sample observations $\left(c_{i}\right)$ are transformed into cumulative binomial values $\left(u_{i}\right)$ using the binomial cumulative distribution function as follows:

$$
u_{i}=F_{B}\left(c_{i} ; n / N_{i}, t_{i}\right) ; i=2,3, \ldots, m
$$

where, $N_{i}$ be the sum of all the sample sizes up to sample $i$ and $t_{i}$ is the sum of all the defects in all the samples up to sample $i$, i.e. $t_{i}=\sum_{j=1}^{i} c_{j}$ and $m$ is the number of samples. It may be noted that $u_{1}$ and $Q_{1}$ cannot be computed while applying the transformation when parameter $\lambda$ is unknown. The cumulative binomial values are retransformed into $Q_{i}(i=2,3, \ldots, m)$ values by using Equation (10).

\subsection{Mapping based approach}

For applying Borges and Ho's (2001) mapping based approach, we need to compute first expected proportion of nonconforming units with respect to $U S L\left(P N U_{U}\right)$ for STB type events and proportion of nonconforming units with respect to $L S L\left(P N U_{L}\right)$ for LTB type events. The estimates of $P N U_{U}$ and $P N U_{L}$ can be obtained as follows:

$$
\begin{aligned}
& \widehat{P N U}_{U}=P\left\{c>c_{U}\right\}=1-P\left\{c \leq c_{U}\right\}=1-\sum_{c=0}^{c_{U}} e^{-\bar{c}}(\bar{c})^{c} / c ! \\
& \widehat{P N U}_{L}=P\left\{c<c_{L}\right\}=\sum_{c=0}^{c_{L}-1} e^{-\bar{c}}(\bar{c})^{c} / c !
\end{aligned}
$$

After obtaining the values of $P N U_{U}$ and $P N U_{L}$, the $C_{p u}$ or $C_{p l}$ of a Poisson process can be estimated in two steps:

1) Determine the corresponding $Z$-value of the standard normal distribution that 'results in probability area equal to $P N U_{U}$ or $P N U_{L}$ on the upper tail. Let the Z-values corresponding to $P N U_{U}$ and $P N U_{L}$ are $Z_{U}$ and $Z_{L}$ respectively. These values can be obtained by using inverse cumulative probability of the standard normal distribution function as follows:

$$
Z_{U}=\Phi^{-1}\left(1-P N U_{U}\right) \text { and } Z_{L}=\Phi^{-1}\left(1-P N U_{L}\right)
$$

where, $\Phi(\cdot)$ denotes the standard normal cumulative distribution function.

2) Obtain the estimates of $C_{p u}$ and $C_{p l}$ as follows:

$$
\begin{aligned}
& \hat{C}_{p u}=\hat{C}_{u}=(1 / 3) \times Z_{U} \\
& \hat{C}_{p l}=\hat{C}_{l}=(1 / 3) \times Z_{L}
\end{aligned}
$$

It must be noted that if $\widehat{P N U}_{U}$ is greater than equal to 0.5 , then $\hat{C}_{u}$ is considered as zero. Similarly, if $\widehat{P N U}_{L}$ is greater than equal to 0.5 , then $\hat{C}_{l}$ is considered as zero.

\subsection{Process nonconforming based approach}

Yeh and Bhattacharya (1998) and Perakis and Xekalaki $(2002,2005)$ proposed indices $C_{f}$ and $C_{p c}$ respectively, and these indices measure the process capability by looking directly at the proportion of nonconforming in a given process. In case of unilateral specification, the $C_{f}$ index can be expressed as

$$
\begin{aligned}
C_{f u} & =\alpha_{0}^{U} / \alpha_{U} \\
C_{f l} & =\alpha_{0}^{L} / \alpha_{L}
\end{aligned}
$$


where, $\alpha_{0}^{U}$ (or $\alpha_{0}^{L}$ ) is the maximum proportion of nonconforming in the upper (lower) side that the manufacturer can tolerate. On the other hand, $\alpha_{U}\left(=\widehat{P N U}_{U}\right)$ is the actual proportion of nonconforming in the upper side, and $\alpha_{L}\left(=\widehat{P N U}_{L}\right)$ is the actual proportion of nonconforming in the lower side. Following the convention for normal distribution, Yeh and Bhattacharya (1998) recommend to consider $\alpha_{0}^{U}=0.00135=\alpha_{0}^{L}$.

For unilateral specification, Perakis and Xekalaki $(2002,2005)$ proposed $C_{p c}$ index can be expressed as

$$
\begin{aligned}
C_{p c u} & =\left(1-p_{0}^{U}\right) /\left(1-p_{U}\right) \\
C_{p c l} & =\left(1-p_{0}^{L}\right) /\left(1-p_{L}\right)
\end{aligned}
$$

where, $p_{0}^{U}$ is the desired proportion of conforming units with respect $U S L$ and $p_{0}^{L}$ is the desired proportion of conforming units with respect to $L S L$. On the other hand, $p_{U}\left(=1-\widehat{P N U}_{U}\right)$ is the actual proportion of conforming units with respect to USL, and $p_{L}\left(=1-\widehat{P N U}_{L}\right)$ is the actual proportion of conforming units with respect to $L S L$. It may be noted that $1-p_{U}=\alpha_{U}\left(=\widehat{P N U}_{U}\right)$ and $1-p_{L}=\alpha_{L}\left(=\widehat{P N U}_{L}\right)$. Perakis and Xekalaki $(2002,2005)$ recommend that 0.9973 is a good choice for the desired proportion of conformance for both sided specifications and thus, a good choice for the desired proportion of conformance for one sided specification is 0.99865 . So, $1-p_{0}^{U}=0.00135=\alpha_{0}^{U}$ and $1-p_{0}^{L}=0.00135=\alpha_{0}^{L}$. Thus, the indices defined by Yeh and Bhattacharya (1998) and Perakis and Xekalaki $(2002,2005)$ are essentially the same in case of unilateral specification.

It can be shown that the index $C_{p c u}$ or $C_{p c l}$ will always be greater than or equal to 0.00135 and it can take very high value when the actual proportion of conformance approaches to 1 .

\subsection{Process yield based approach}

Maity et al. (2010) proposed $C_{p y}$ index as a measure of process capability. For a quality characteristic with both sided specifications, the $C_{p y}$ index is calculated as follows:

$$
C_{p y}=\frac{[F(U)-F(L)]}{\left[1-\alpha_{0}^{U}-\alpha_{0}^{L}\right]}
$$

where $F(U)$ and $F(L)$ are cumulative probability distribution function of the quality characteristic at $U S L$ and $L S L$ respectively, and $\alpha_{0}^{U}$ and $\alpha_{0}^{L}$ are the maximum allowable proportion of nonconforming at upper tail and lower tail of the distribution respectively. Here the numerator, $F(U)-F(L)$, gives the measure of the actual process yield (i.e. actual proportion of conforming) and the denominator, $\left(1-\alpha_{0}^{U}-\alpha_{0}^{L}\right)$ gives the measure of the desired process yield (i.e. desired proportion of conforming).

Maiti et al. (2010) suggested that in case of unilateral specification, median of the distribution $\left(\mu_{e}\right)$ should be taken as the process target and the process centre should be located such that $F\left(\mu_{e}\right)=[F(U)+F(L)] / 2=1 / 2=0.5$. Therefore, for unilateral specification, $C_{p y}$ index can be expressed as

$$
\begin{aligned}
& C_{p y u}=\frac{F(U)-F\left(\mu_{e}\right)}{1-\alpha_{0}^{U}-F\left(\mu_{e}\right)}=\frac{F(U)-0.5}{0.5-\alpha_{0}^{U}} \\
& C_{p y l}=\frac{F\left(\mu_{e}\right)-F(L)}{F\left(\mu_{e}\right)-\alpha_{0}^{L}}=\frac{0.5-F(L)}{0.5-\alpha_{0}^{L}}
\end{aligned}
$$

where the value of $\alpha_{0}^{U}$ or $\alpha_{0}^{L}$ is conventionally taken as 0.00135 .

It can easily be verified that the index $C_{p y u}$ or $C_{p y l}$ can take a maximum value of 1.0027 . Also, if $F(U)$ is less than or equal to 0.5 , then $C_{p y u}$ is considered as zero. Similarly, if $F(L)$ is greater than or equal to 0.5 , then $C_{p y l}$ is considered as zero.

\section{Procedure for assessing relative goodness of the estimated $\widehat{C}_{p u}\left(\right.$ or $\left.\widehat{C}_{p l}\right)$ values obtained by different methods}

The importance of process capability index lies in the fact that the analysts/users can predict the extent of product conformance to the specifications by examining the estimated value of the index, and accordingly he/she can take appropriate decision. For example, if a product characteristic $X$ follows normal distribution and $\hat{C}_{p u}=1$, then it implies that expected proportion of conforming products (EPCP) in the process outputs with respect to the $U S L$ of $X\left(s a y, U S L_{X}\right)$ is 0.99865 , i.e. the process is capable of producing $99.865 \%$ conforming products. This interpretation is derived from the following relationship:

$$
E P C P=P\left(X \leq U S L_{X}\right)=P\left(\frac{X-\widehat{\mu}}{\widehat{\sigma}} \leq \frac{U S L_{X}-\widehat{\mu}}{\widehat{\sigma}}\right)=P\left(z \leq 3 \times \frac{U S L_{X}-\widehat{\mu}}{3 \widehat{\sigma}}\right)=P\left(z \leq 3 \times \hat{C}_{p u}\right)=\Phi\left(3 \times \hat{C}_{p u}\right)
$$


where $\hat{\mu}$ and $\hat{\sigma}$ are the estimates of mean and standard deviation of $X$, respectively.

Similarly, it is desired that the analysts/users will be able to predict the expected proportion of conforming units (EPCU) in a Poisson process by examining the estimated value of the process capability index. However, the count data $(c)$ in an inspection unit does not follow normal distribution, but follows Poisson distribution, and thus, Equation (23) is not applicable for predicting EPCU in a Poisson process. It may be recalled that among various methods discussed in Section 2.0, only in mapping based approach (Borges and Ho, 2001) the proportion of nonconforming units in the Poisson process is mapped to the Z-value of the standard normal distribution that results in the same proportion of nonconformance, and then process capability index with respect to USL (or LSL), denoted as $\hat{C}_{u}$ (or $\hat{C}_{l}$ ), is estimated as one-third of the mapped Z-value. Thus, it is possible to predict the EPCU in a Poisson process by examining the estimated $\hat{C}_{u}$ (or $\hat{C}_{l}$ ) value, which is feasible due to the following relationship:

$$
\begin{aligned}
& \frac{1}{3} \times Z_{U}=\hat{C}_{u} \\
& \Rightarrow \quad Z_{U}=3 \hat{C}_{u} \\
& \Rightarrow \quad \Phi^{-1}\left(1-P N U_{U}\right)=3 \hat{C}_{u} \\
& \Rightarrow \quad\left(1-P N U_{U}\right)=\Phi\left(3 \hat{C}_{u}\right) \\
& \Rightarrow \quad E P C U=\Phi\left(3 \hat{C}_{u}\right)
\end{aligned}
$$

Equations (23) and (24) reveal that equal values of estimated $\hat{C}_{u}$ from a Poisson process and estimated $\hat{C}_{p u}$ from a normal process bear the same interpretation. For example, $\hat{C}_{p u}=1$ will imply that the normal process is capable of producing $99.865 \%$ conforming products with respect to the $U S L$ of $X\left(\right.$ say, $U S L_{X}$ ) and $\hat{C}_{u}=1$ will also imply that the Poisson process is capable of producing $99.865 \%$ conforming units, i.e. units having number of defects less than the specified $U S L$ (say, $c_{U}$ ).

From the above discussions, it is clear that if the process capability index of a Poisson process with respect to USL is measured in terms of $C_{u}$, i.e. if $\hat{C}_{p u}=\hat{C}_{u}$, then $\hat{C}_{p u}$ value gives the most appropriate measure of capability of a Poisson process with respect to $U S L$ in the sense that the analysts/users can easily comprehend the truly expected percentage of conforming units in the Poisson process by examining the $\hat{C}_{p u}=\hat{C}_{u}$ value. Similarly, if $C_{p l}$ of a Poisson process is estimated in terms of $C_{l}$, i.e. $\hat{C}_{p l}=\hat{C}_{l}$, then $\hat{C}_{p l}$ value gives the most appropriate measure of capability of a Poisson process with respect to $L S L$. Therefore, goodness of any other estimates of $C_{p u}$ (or $C_{p l}$ ) may be judged by comparing the estimated values with the $\hat{C}_{u}$ (or $\hat{C}_{l}$ ) value.

Estimation of confidence interval of $\hat{C}_{u}$

Since $\hat{C}_{u}$ is a point estimate obtained from sample data, it is necessary to construct confidence interval (CI) of the capability index $C_{u}$ for inference purpose, especially when the sample size is relatively small. In literature, many works (Chou et al., 1990; Zhang et al., 1990; Kushler and Hurley, 1992; Nagata, 1991; Nagata and Nagahata, 1994; Peng, 2010; Stoma et al., 2019) are reported on the construction of confidence intervals of estimated capability indices. But all these works assume that quality characteristic of interest follows normal distribution. To the best of our knowledge, no work is reported in literature on construction of confidence intervals of the process capability indices estimated from a Poisson process. Hence, we use Nagata and Nagahata (1994) proposed approximation formula for construction of two-sided confidence interval (CI) for $\hat{C}_{u}$. According to Nagata and Nagahata (1994),

$$
(1-\alpha) \% \text { two-sided CI of } \hat{C}_{u}=\left(\hat{C}_{u}-Z_{1-\frac{\alpha}{2}} \sqrt{\frac{1}{9 n}+\frac{\hat{C}_{u}^{2}}{2(n-1)}}, \hat{C}_{u}+Z_{1-\frac{\alpha}{2}} \sqrt{\frac{1}{9 n}+\frac{\hat{C}_{u}^{2}}{2(n-1)}}\right)
$$

where, $\alpha$ is the level of significance and $(1-\alpha)$ is the confidence coefficient.

\section{Assessing relative goodness of the estimates of $C_{p u}\left(\right.$ or $\left.C_{p \nu}\right)$ obtained by different approaches}

It is decided to compute first $\hat{C}_{u}$ value from a set of observed count data and determine its $95 \%$ confidence interval (CI), and then to compute $\hat{C}_{p u}$ values using all other approaches from the same set of observed count data. As it is mentioned earlier that $\hat{C}_{p u}=$ $\hat{C}_{u}$ value gives the most appropriate measure of capability of a Poisson process, and therefore, the goodness of estimated $\hat{C}_{p u}$ values obtained by other methods are judged based on the following two considerations:

i) If the estimated $\hat{C}_{p u}$ value obtained by a method lie within the $95 \%$ CI of $C_{u}$ or not.

ii) Percent deviation of an estimated $\hat{C}_{p u}$ value with respect to $\hat{C}_{u}$ value, and it is calculated as follows:

$$
\text { Percent deviation }=\frac{\hat{C}_{p u}-\hat{C}_{u}}{\hat{C}_{u}} \times 100
$$




\section{Analysis and related results}

For the purpose of analysis, the following three data sets are selected arbitrarily - (i) case study data published in Maravelakis (2016), (ii) process data presented in Montgomery (2009, pp. 698) and (iii) process data presented in NIST/SEMATECH eHandbook of Statistical Methods (2012, section 6.3.3.1).

\subsection{Case study data in Maravelakis (2016)}

Maravelakis (2016) considered a manufacturing process for illustrating his proposed transformation approach for process capability index. In this case study, 100 units of products were collected and number of occurrences of defects on each product was recorded. The USL for the number of defects in a unit was $c_{U}=9$.

In the data set, the total number of defects is found to be $\sum_{i=1}^{100} c_{i}=506$. So, unknown parameter $\lambda$ is estimated as $\hat{\lambda}=\bar{c}=$ $506 / 100=5.06$. The defects data $c_{i}(i=1,2,3, \ldots, 100)$ are plotted in a $c$-chart, which revealed that during sample collection the process was in control. These data are analyzed first using mapping based approach (Borges and Ho, 2001) for obtaining the point estimate of $C_{u}$ and $95 \%$ both sided confidence interval of $C_{u}$.

Here, $\hat{\lambda}=\bar{c}=5.06$ and $c_{U}=9$. So $P N U_{U}$ and $Z_{U}$ values are obtained as follows:

$$
\begin{aligned}
P N U_{U} & =1-\sum_{c=0}^{9} e^{-5.06}(5.06)^{c} / c !=1-0.96594=0.03406 \\
Z_{U} & =\Phi^{-1}\left(1-P N U_{U}\right)=\Phi^{-1}(1-0.03406)=\Phi^{-1}(0.96594)=1.824
\end{aligned}
$$

Thus, $C_{u}$ is estimated as

$$
\hat{C}_{u}=(1 / 3) \times Z_{U}=(1 / 3) \times 1.824=0.608
$$

Using Nagata and Nagahata's (1994) approximate formula, 95\% confidence interval (CI) of $C_{u}$ is obtained as follows:

$$
\begin{aligned}
& {\left[\hat{C}_{u}-Z_{1-\frac{\alpha}{2}} \sqrt{\frac{1}{9 n}+\frac{\hat{C}_{u}^{2}}{2(n-1)}}, \quad \hat{C}_{u}+Z_{1-\frac{\alpha}{2}} \sqrt{\frac{1}{9 n}+\frac{\hat{C}_{u}^{2}}{2(n-1)}}\right]} \\
& \Rightarrow\left[0.608-1.96 \times \sqrt{\frac{1}{9 \times 100}+\frac{0.608^{2}}{2(100-1)}}, 0.608+1.96 \times \sqrt{\frac{1}{9 \times 100}+\frac{0.608^{2}}{2(100-1)}}\right] \\
& \Rightarrow[0.5011,0.7151]
\end{aligned}
$$

From the above analysis it is found that the most appropriate measure of process capability with respect to USL in the Maravelakis (2016) considered Poisson process is 0.608 and $95 \%$ CI of the process capability of this process is [0.5011, 0.7151]. All other five approaches, described in section 2, are now applied to the same data of Maravelakis (2016) for estimating $C_{p u}$ values.

Estimation of $C_{p u}$ using normal approximation approach

Here $\hat{\lambda}=\bar{c}=5.06$, which is greater than 5 . Therefore, using normal approximation approach $C_{p u}$ is estimated as

$$
\hat{C}_{p u}=\left(c_{U}-\bar{c}\right) /(3 \times \sqrt{\bar{c}})=(9-5.06) /(3 \times \sqrt{5.06})=0.5838
$$

Estimation of $C_{p u}$ using percentile based approach

The $50^{\text {th }}$ percentile point (i.e. median $\left.M\right)$ and the $99.865^{\text {th }}$ percentile point $\left(C_{0.99865}\right)$ for the Poisson distribution with $\hat{\lambda}=\bar{c}=5.06$ are found to be 5 and 13 respectively. Thus, $C_{p u}$ is estimated as

$$
\hat{C}_{p u}=\left(c_{U}-M\right) /\left(C_{0.99865}-M\right)=(9-5) /(13-5)=0.5
$$

Estimation of $C_{p u}$ using transformation approach

Here, $\hat{\lambda}=\bar{c}=5.06$ and $U S L=c_{\mathrm{U}}=9$. The observed defects data $c_{i}(i=1,2,3, \ldots, 100)$ are transformed into cumulative Poisson values $u_{i}$ assuming that $\lambda=5.06$ using Equation (9). The $c_{\mathrm{U}}=9$ was also transformed into $Q_{U}$ value as follows:

$$
Q_{U}=\Phi^{-1}\left\{F_{P}(9 \mid \lambda=5.06)\right\}=\Phi^{-1}\{0.96817\}=1.82426
$$


The average value $\bar{Q}$ and $S D_{Q}$ are found to be 0.2456 and 0.8263 respectively. Thus, the process capability index $C_{p u}$ is estimated as

$$
\hat{C}_{p u}=\frac{Q_{U}-\bar{Q}}{3 \times S D_{Q}}=\frac{1.82426-0.2456}{3 \times 0.8263}=0.6368
$$

Estimation of $C_{p u}$ using process nonconforming based approach

The desired proportion of conforming units with respect $U S L, p_{0}^{U}=P C U_{U}$ is not specified and so as per convention it is taken as 0.99865. This implies that $\left(1-p_{0}^{U}\right)=\left(1-P C U_{U}\right)=0.00135$. Here, $\hat{\lambda}=\bar{c}=5.06$, and the actual proportion of nonconforming units, $\left(1-p_{U}\right)=\left(1-\widehat{P N U}_{U}\right)$ is computed to be 0.03406 . Therefore, the process capability index $C_{p u}$ is estimated as

$$
\hat{C}_{p u}=\hat{C}_{p c u}=0.00135 / 0.03406=0.0396
$$

Estimation of $C_{p u}$ using process yield based approach

Here, $\hat{\lambda}=\bar{c}=5.06$ and $c_{\mathrm{U}}=9$. As per convention, the maximum allowable proportion of nonconforming units at upper tail, $\alpha_{0}^{U}$ is considered to be 0.00135 . The cumulative probability up to the $U S L, F(U)$ is computed as 0.96594 . Therefore, $C_{p u}$ is estimated as

$$
\hat{C}_{p u}=\hat{C}_{p y u}=(0.96594-0.5) /(0.5-0.00135)=0.46594 / 0.49865=0.9344
$$

It may be noted that estimated $\hat{C}_{p u}$ values obtained by the five approaches varies widely. Now, for the purpose of easy comparison

\begin{tabular}{|c|c|c|c|c|c|}
\hline $\begin{array}{l}\text { Sl. } \\
\text { No. }\end{array}$ & $\begin{array}{c}\hat{C}_{u} \\
\left(95 \% \text { CI of } C_{u}\right)\end{array}$ & $\begin{array}{l}\text { Approaches for } C_{p u} \\
\text { calculation }\end{array}$ & $\begin{array}{c}\text { Estimated } \hat{C}_{p u} \\
\text { value }\end{array}$ & $\begin{array}{c}\hat{C}_{p u} \text { value in reference } \\
\text { to the CI of } C_{u}\end{array}$ & $\begin{array}{l}\text { Percent } \\
\text { deviation }\end{array}$ \\
\hline 1 & \multirow{5}{*}{$\begin{array}{c}0.6081 \\
(0.5011-0.7151)\end{array}$} & $\begin{array}{l}\text { Normal approx. } \\
\text { approach }\end{array}$ & 0.5838 & Within CI & -3.98 \\
\hline 2 & & $\begin{array}{l}\text { Percentile based } \\
\text { approach }\end{array}$ & 0.5000 & $\begin{array}{c}\text { Below lower } \\
\text { confidence limit }\end{array}$ & -17.76 \\
\hline 3 & & $\begin{array}{l}\text { Transformation } \\
\text { approach }\end{array}$ & 0.6368 & Within CI & +4.73 \\
\hline 4 & & $\begin{array}{l}\text { Nonconforming } \\
\text { based approach }\end{array}$ & 0.0396 & $\begin{array}{c}\text { Below lower } \\
\text { confidence limit }\end{array}$ & -93.49 \\
\hline 5 & & $\begin{array}{l}\text { Yield-based } \\
\text { approach }\end{array}$ & 0.9344 & $\begin{array}{c}\text { Above upper } \\
\text { confidence limit }\end{array}$ & +53.68 \\
\hline
\end{tabular}
of goodness of these estimated $\hat{C}_{p u}$ values, Table 1 is prepared.

Table 1: $\hat{C}_{u}, 95 \% \mathrm{CI}$ of $C_{u}$ and the $\hat{C}_{p u}$ values obtained by other approaches

Table 1 reveals that only the estimated $\hat{C}_{p u}$ values obtained by Normal approximation approach and Transformation approach lie within the $95 \% \mathrm{CI}$ of $\hat{C}_{u}$. On the other hand, estimated $\hat{C}_{p u}$ values obtained by Percentile based approach and Nonconforming based approach lie below the lower confidence limit of the CI whereas estimated $\hat{C}_{p u}$ values obtained by Yield based approach lies above the upper confidence limit of the CI. This implies that Percentile based approach and Nonconforming based approach underestimate the process capability (deviations are $-17.76 \%$ and $-93.49 \%$ respectively) whereas Yield based approach overestimate the process capability (deviation is $+53.68 \%$ ) for the Poisson process.

\subsection{Process data in Montgomery (2009)}

Montgomery (2009) presents a set of process data on number of defects observed on 20 inspection units of printed circuit boards (PCBs) for illustration of attribute process control charts. Each inspection unit consists of successive samples of five PCBs and each inspection unit is collected after every one hour. The total number of defects is found to be 160 and therefore, the estimate of the unknown parameter is obtained as $\hat{\lambda}=\bar{c}=160 / 20=8$. The $c$-chart of the defects data indicates that the process was in statistical control during sample collection.

Montgomery (2009) did not specify USL for the number of defects in an inspection unit of PCBs. For the purpose of estimation of $C_{p u}$, we assume that $\bar{c}+2 \times \sqrt{c}=8+5.66 \approx 14$ is the $U S L$ for the number of defects in an inspection unit, i.e. $c_{U}=14$. These data are analyzed first using mapping based approach for obtaining the point estimate and $95 \%$ both sided CI of $C_{u}$. 
It is found that $\hat{C}_{u}=0.7047$ and $95 \% \mathrm{CI}$ of $C_{u}=[0.4373,0.9721]$. This implies that the most appropriate estimate of process capability with respect to USL of the considered Poisson process is 0.7047 , and its $95 \%$ CI is $[0.4373,0.9721]$. The $\hat{C}_{p u}$ values are now obtained by applying all other five approaches to the same data of Montgomery (2009). The estimated $\hat{C}_{p u}$ values obtained by other five approaches along with $\hat{C}_{u}$ value and $95 \%$ CI of $C_{u}$ are presented in Table 2 .

Table 2: $\hat{C}_{u}, 95 \%$ CI of $C_{u}$ and the $\hat{C}_{p u}$ values obtained by other approaches

\begin{tabular}{|c|c|c|c|c|c|}
\hline $\begin{array}{l}\text { Sl. } \\
\text { No. }\end{array}$ & $\begin{array}{c}\hat{C}_{u} \\
\left(95 \% \text { CI of } C_{u}\right)\end{array}$ & $\begin{array}{c}\text { Approaches for } C_{p u} \\
\text { calculation }\end{array}$ & $\begin{array}{l}\text { Computed } \\
\hat{C}_{p u} \text { value }\end{array}$ & $\begin{array}{c}\hat{C}_{p u} \text { value in reference } \\
\text { to the CI of } C_{u}\end{array}$ & $\begin{array}{l}\text { Percent } \\
\text { deviation }\end{array}$ \\
\hline 1 & \multirow{5}{*}{$\begin{array}{c}0.7047 \\
(0.4372-0.9621)\end{array}$} & $\begin{array}{l}\text { Normal approx. } \\
\text { approach }\end{array}$ & 0.7071 & Within CI & +0.34 \\
\hline 2 & & $\begin{array}{l}\text { Percentile based } \\
\text { approach }\end{array}$ & 0.6000 & Within CI & -14.86 \\
\hline 3 & & $\begin{array}{l}\text { Transformation } \\
\text { approach }\end{array}$ & 0.4416 & Within CI & -37.33 \\
\hline 4 & & $\begin{array}{l}\text { Nonconforming } \\
\text { based approach }\end{array}$ & 0.0782 & $\begin{array}{c}\text { Below lower } \\
\text { confidence limit }\end{array}$ & -88.90 \\
\hline 5 & & $\begin{array}{l}\text { Yield-based } \\
\text { approach }\end{array}$ & 0.9681 & $\begin{array}{c}\text { Above upper } \\
\text { confidence limit }\end{array}$ & +37.38 \\
\hline
\end{tabular}

Table 2 shows that the estimated $\hat{C}_{p u}$ values obtained by Normal approximation approach, Percentile based approach and Transformation approach lie within the $95 \%$ CI of $C_{u}$. On the other hand, estimated $\hat{C}_{p u}$ value obtained by Nonconforming based approach lies below the lower confidence limit of the CI whereas estimated $\hat{C}_{p u}$ value obtained by Yield based approach lies above the upper confidence limit of the CI. This implies that Nonconforming based approach underestimate the capability (deviations is $88.90 \%$ ) whereas Yield based approach overestimate the capability (deviation is $+37.38 \%$ ) of the Poisson process.

\subsection{Process data in NIST/SEMATECH e-Handbook of Statistical Methods (2012)}

The NIST/SEMATECH e-Handbook of Statistical Methods (2012) presents inspection results on 25 wafers each containing 100 chips for illustrating construction of $c$-chart. The total number of defects is found to be 400 and therefore, the estimate of the unknown parameter is obtained as $\hat{\lambda}=\bar{c}=400 / 25=16$. The $c$-chart of the defects data indicates that the process was in statistical control during sample collection. Here, USL for the number of defects in a wafer is not specified. So, we assume that $\bar{c}+$ $2 \times \sqrt{c}=16+2 \sqrt{16}=24$ is the $U S L$ for the number of defects in a wafer, i.e. $c_{U}=24$. These data are analyzed first using mapping based approach for obtaining the point estimate of $C_{u}$ and $95 \%$ both sided CI of $C_{u}$.

It is found that $\hat{C}_{u}=0.6694$ and $95 \%$ CI of $C_{u}=[0.4393,0.8994]$. The $\hat{C}_{p u}$ values are now estimated by applying all other five approaches to the same data. The estimated $\hat{C}_{p u}$ values obtained by other five approaches along with $\hat{C}_{u}$ value and $95 \% \mathrm{CI}$ of $C_{u}$ are presented in Table 3.

Table 3: $\hat{C}_{u}, 95 \%$ CI of $C_{u}$ and the $\hat{C}_{p u}$ values obtained by other approaches

\begin{tabular}{|c|c|c|c|c|c|}
\hline $\begin{array}{l}\text { Sl. } \\
\text { No. }\end{array}$ & $\begin{array}{c}\hat{C}_{u} \\
\left(95 \% \text { CI of } C_{u}\right)\end{array}$ & $\begin{array}{c}\text { Approaches for } C_{p u} \\
\text { calculation }\end{array}$ & $\begin{array}{l}\text { Computed } \\
\hat{C}_{p u} \text { value }\end{array}$ & $\begin{array}{c}\hat{C}_{p u} \text { value in reference to } \\
\text { the CI of } C_{u}\end{array}$ & $\begin{array}{l}\text { Percent } \\
\text { deviation }\end{array}$ \\
\hline 1 & \multirow{5}{*}{$\begin{array}{c}0.6694 \\
(0.4393-0.8994)\end{array}$} & $\begin{array}{l}\text { Normal approx. } \\
\text { approach }\end{array}$ & 0.6667 & Within CI & -0.40 \\
\hline 2 & & $\begin{array}{l}\text { Percentile based } \\
\text { approach }\end{array}$ & 0.6154 & Within CI & -8.07 \\
\hline 3 & & $\begin{array}{l}\text { Transformation } \\
\text { approach }\end{array}$ & 0.6616 & Within CI & -1.16 \\
\hline 4 & & $\begin{array}{l}\text { Nonconforming } \\
\text { based approach }\end{array}$ & 0.0601 & $\begin{array}{c}\text { Below lower } \\
\text { confidence limit }\end{array}$ & -91.03 \\
\hline 5 & & $\begin{array}{l}\text { Yield-based } \\
\text { approach }\end{array}$ & 0.9580 & $\begin{array}{c}\text { Above upper } \\
\text { confidence limit }\end{array}$ & +43.11 \\
\hline
\end{tabular}

Table 3 reveals that the estimated $\hat{C}_{p u}$ values obtained by Normal approximation approach, Percentile based approach and Transformation approach lie within the $95 \%$ CI of $C_{u}$. However, estimated $\hat{C}_{p u}$ value obtained by Nonconforming based approach lies below the lower confidence limit of the CI and estimated $\hat{C}_{p u}$ value obtained by Yield based approach lies above the upper 
confidence limit of the CI. This implies that Nonconforming based approach underestimate the capability (deviations is $-91.03 \%$ ) whereas Yield based approach overestimate the capability (deviation is $+43.11 \%$ ) of the Poisson process.

The overall results of the three case study data analysis can be summarized as follows:

a) The Nonconforming based approach always underestimates the capability of a Poisson process. The estimated $\hat{C}_{p u}$ values obtained by this approach in the three case studies deviate from the most appropriate measures of process capability by $88.90 \%$ to $-93.49 \%$.

b) The Yield based approach always overestimates the capability of a Poisson process. The estimated $\hat{C}_{p u}$ values obtained by this approach in the three case studies deviate from the most appropriate measures of process capability by $+37.38 \%$ to $+53.68 \%$.

c) The estimated $\hat{C}_{p u}$ values obtained by Percentile based approach may or may not lie within the $95 \% \mathrm{CI}$ of $C_{u}$. The estimated $\hat{C}_{p u}$ value in the first case study lies below the lower confidence limit of the CI, but the estimated $\hat{C}_{p u}$ values in the second and third case studies lie within the CI. The estimated $\hat{C}_{p u}$ values obtained by this approach in the three case studies deviate from the most appropriate measures of process capability by $-17.76 \%$ to $-8.07 \%$. This is indicative that Percentile based approach tends to underestimate the capability of a Poisson process.

d) The estimated $\hat{C}_{p u}$ values obtained by Normal approximation approach and Transformation approach always lie within the $95 \%$ CI of $C_{u}$, and therefore, these two approaches can be considered as acceptable for measuring capability of a Poisson process. However, among these two approaches, Normal approximation approach is preferable because the estimated $\hat{C}_{p u}$ value obtained by this approach in the three case studies deviates marginally $(-3.98 \%$ to $+0.34 \%)$ from the most appropriate measures of process capability.

\section{Discussions}

It is observed that equal values of estimated $\hat{C}_{u}$ (or $\hat{C}_{l}$ ) from a Poisson process and estimated $\hat{C}_{p u}\left(\right.$ or $\left.\hat{C}_{p l}\right)$ from a normal process bear the same interpretation. For example, $\hat{C}_{u}=1$ implies that the Poisson process is capable of producing $99.865 \%$ conforming units with respect to the $U S L$, and $\hat{C}_{p u}=1$ implies that the normal process is capable to producing $99.865 \%$ conforming products with respect to the USL. Thus, it is considered that the most appropriate measure of capability of a Poisson process with respect to $U S L$ (or $L S L$ ) is $\hat{C}_{u}$ (or $\hat{C}_{l}$ ). Therefore, the relative goodness of $\hat{C}_{p u}$ values that can be obtained from a Poisson process by other methods are judged with reference to $\hat{C}_{u}$ value and $95 \%$ CI of $C_{u}$ estimated from the observed count data in the Poisson process.

It is observed that the estimated $\hat{C}_{p u}$ values obtained by Nonconforming based approach in the three case studies deviate from the estimated $\hat{C}_{u}$ values by $-88.90 \%$ to $-93.49 \%$. On the other hand, the estimated $\hat{C}_{p u}$ values obtained by Yield-based approach in the three case studies deviate from the estimated $\hat{C}_{u}$ values by $+37.38 \%$ to $+53.68 \%$. The problem with the Nonconforming based approach is that in this approach, $C_{p u}$ (or $C_{p l}$ ) is estimated as the ratio of the two very small numbers, where numerator is 0.00135 (acceptable proportion of nonconforming units) and denominator is actual proportion of nonconforming units with respect to specified $U S L$ (or $L S L$ ). Thus, the estimate is highly impacted due to a minor deviation in the value of actual proportion from the acceptable proportion. For example, if actual percentage of nonconforming units is $0.135 \%$ then the value of $\hat{C}_{p u}$ is equal to one but if the actual percentage becomes $0.01 \%$ then the value of $\hat{C}_{p u}$ would become as high as 13.5 , which would give a misleading impression that the process is highly capable. On the other hand, if the actual percentage becomes $0.5 \%$ then the value of $\hat{C}_{p u}$ would become as low as 0.27 , which again gives a misleading impression that the process capability is very poor.

The Yield-based approach suffers from another problem. In Yield-based approach, the values of the ratios $[F(U)-0.5] /\left(0.5-\alpha_{0}^{U}\right)$ and $[0.5-F(L)] /\left(0.5-\alpha_{0}^{L}\right)$ are considered as the estimate of $C_{p u}$ and $C_{p l}$ respectively. Since the values $\alpha_{0}^{U}$ and $\alpha_{0}^{L}$ are usually taken as 0.00135 , the denominator is always equal to 0.49865 in both the ratios. On the other hand, the values of the numerators in both the ratios can be at most 0.5 . Therefore, the maximum value of $\hat{C}_{p u}$ or $\hat{C}_{p l}$ in a process can be $0.5 / 0.49865=1.0027$. This implies that the estimated $\hat{C}_{p u}$ or $\hat{C}_{p l}$ obtained by Yield-based approach would fail to make distinction among almost capable process, just capable process and highly capable process.

The estimated $\hat{C}_{p u}$ value obtained by Percentile based approach is found to lie below the lower confidence limit of the CI of $C_{u}$ in the first case study, but the estimated $\hat{C}_{p u}$ values obtained by Percentile based approach in the other two case studies are found to lie within the CIs. The estimated $\hat{C}_{p u}$ values obtained by this approach in the three case studies deviate from the estimated $\hat{C}_{u}$ values by $-17.76 \%$ to $-8.07 \%$. These results are indicative that an estimated $\hat{C}_{p u}$ value obtained by Percentile based approach may or may not reflect the capability of a Poisson process appropriately. This happens because the Poisson data is discrete due to which it is not possible to find out the exact $50^{\text {th }}, 99.865^{\text {th }}$ or $0.135^{\text {th }}$ percentile points. The actual percentile values corresponding to the nearest integers may often differ substantially and as a result, the estimated $\hat{C}_{p u}$ or $\hat{C}_{p l}$ value may become considerably different from the true process capability. Therefore, use of Percentile based approach should be avoided for estimating $C_{p u}$ or $C_{p l}$ of a Poisson process. 
The estimated $\hat{C}_{p u}$ values obtained by Normal approximation approach and Transformation approach consistently lie within the 95\% CI of $C_{u}$ in the three case studies, and therefore, these two approaches may be considered as acceptable for measuring capability of a Poisson process besides the Mapping based approach of Borges and Ho (2001). However, among these two approaches, Normal approximation approach is preferable because the estimated $\hat{C}_{p u}$ values obtained by this approach in the three case studies deviates marginally $(-3.98 \%$ to $+0.34 \%)$ from the most appropriate measure, i.e. $\hat{C}_{u}$. It must be noted that above conclusion is valid only when average number of defects per inspection unit is more than 5 because in all the three case study data analyzed here $\bar{c}>5$.

It is worth to mention here some limitations of the current study. In this study, the relative goodness of the process capability indices obtained by different approaches is assessed primarily based on the results of analysis of multiple case study data published in literature. It is well known that accuracy of the estimate of population parameter $\lambda$ and thus, estimates of process capability indices depends on the sample size. Here Poisson data with three different sample sizes only are analysed and so, ideally, the findings of this study should not be generalized. Simulation of Poisson data of different sizes and then application of the similar studies will be useful for generalization of the findings.

\section{Conclusions}

In real life, often number of occurrences of some events in an inspection unit is considered as an important quality characteristic. Such quality characteristics are usually STB or LTB type and often are modeled by Poisson distribution with parameter $\lambda(>0)$. In such cases, computation of process capability index $\left(C_{p u}\right.$ or $\left.C_{p l}\right)$ using standard formula is inappropriate. Usually, $C_{p u}$ or $C_{p l}$ of a Poisson process is estimated using some approximate approaches or generalized approaches. It is observed that the most appropriate measure of capability of a Poisson process with respect to $U S L$ (or $L S L$ ) is $\hat{C}_{u}$ (or $\hat{C}_{l}$ ) (obtained by Mapping based approach). Therefore, the relative goodness of estimated $\hat{C}_{p u}$ values obtained from a Poisson process by other methods are judged with reference to estimated $\hat{C}_{u}$ value and $95 \%$ confidence interval of $C_{u}$. Analyses of multiple case study data reveal that besides Mapping based approach only Normal approximation approach and Transformation approach are acceptable for estimating $C_{p u}$ or $C_{p l}$ of a Poisson process. On the other hand, Nonconforming based approach usually underestimate the capability whereas Yieldbased approach usually overestimate the capability of a Poisson process. The estimated $\hat{C}_{p u}$ (or $\hat{C}_{p l}$ ) value obtained by Percentile based approach may or may not reflect the true capability of a Poisson process.

In this study, only the Poisson processes where a single type of defect occurs with rate $\lambda$ are considered. In future study, Poisson processes where multiple types of defects occur with different rates may be considered. Zero inflated Poisson process may also be considered in the future study.

\section{Acknowledgement}

We are sincerely grateful to the referees for their valuable comments which have helped in improving the overall clarity and readability of this article.

\section{References}

Ahmad, S., Alatefi, M., Alkahtani, M., Anwar, S., Sharaf, M. \& Abdollahian, M. 2019. Bibliometric analysis for process capability research. Quality Technology \& Quantitative Management, Vol. 16, No. 4, pp. 459-477. https://doi.org/10.1080/16843703.2018.1464426

Borges, W. \& Ho, L.L., 2001. A fraction defective based capability index. Quality and Reliability Engineering International, Vol. 17, No. 6, pp. 447-458. https://doi.org/10.1002/qre.438

Chen, J.P., 2000. Re-evaluating the process capability indices for non-normal distributions. International Journal of Production Research, Vol. 38, No. 6, pp. 1311-1324. https://doi.org/10.1080/002075400188861

Chen, K.S., Huang, M.L. \& Hung, Y.H., 2008. Process capability analysis chart with the application of $C_{p m}$. International Journal of Production Research, Vol. 46, No. 16, pp. 4483-4499. https://doi.org/10.1080/00207540600806422

Chen, K.S., Wang, K.J. \& Chang, T.C., 2017. A novel approach to deriving the lower confidence limit of indices $C_{p u}, C_{p l}$, and $C_{p k}$ in assessing process capability. International Journal of Production Research, Vol. 55, No. 17, pp. 4963-4981. https://doi.org/10.1080/00207543.2017.1282644

Chen, P., Wang, B.X. \& Ye, Z-S. 2019. Yield-based process capability indices for nonnormal continuous data. Journal of Quality Technology, Vol. 5, No. 2, pp. 171-180. https://doi.org/10.1080/00224065.2019.1571342

Clements, J.A., 1989. Process capability calculations for non-normal distributions. Quality Progress, Vol. 22, No. 9, pp. 95-100.

Chou, Y.M, Owen, D.B. \& Borrego, S.A., 1990. Lower confidence limits on process capability indices. Journal of Quality Technology, Vol. 22, No. 3, pp. 223-229. https://doi.org/10.1080/00224065.1990.11979242 
English, J.R. \& Taylor, G.D., 1993. Process capability analysis - a robustness study. International Journal of Production Research, Vol. 31, No. 7, pp. 1621-1635. https://doi.org/10.1080/00207549308956813

Goswami, A. \& Dutta, H.N., 2013. Some studies on normal and non-normal process capability indices. International Journal of Mathematics and Statistics Invention, Vol. 1, No. 2, pp. 31-40.

Grau, D., 2012. Process yield, process centering and capability indices for one-sided tolerance processes. Quality Technology \& Quantitative Management, Vol. 9, No. 2, pp. 153-170. https://doi.org/10.1080/16843703.2012.11673283

Kane, V.E., 1986. Process capability indices. Journal of Quality Technology, Vol. 18, No. 1, pp. 41-52.

Kotz, S. \& Johnson, N.L., 1993. Process Capability Indices. London: Chapman and Hall.

Kotz, S. \& Johnson, N.L, 2002. Process capability indices - a review, 1992-2000. Journal of Quality Technology, Vol. 34, No. 1, pp. 2-19. https://doi.org/10.1080/00224065.2002.11980119

Kushler, R. \& Hurley, P., 1992. Confidence bounds for capability indices. Journal of Quality Technology, Vol. 24, No. 2, pp. 188195. https://doi.org/10.1080/00224065.1992.11979400

Maiti, S.S., Saha, M. \& Nanda, A.K., 2010. On generalising process capability indices. Quality Technology \& Quantitative Management, Vol. 7, No. 3, pp. 279-300. https://doi.org/10.1080/16843703.2010.11673233

Maravelakis, P.E., 2016. Process capability indices for data following the Poisson or Binomial distribution. Quality Technology \& Quantitative Management, Vol. 13, No. 2, pp. 197-206. https://doi.org/10.1080/16843703.2016.1169688

Montgomery, D.C., 2009. Introduction to Statistical Process Control, 6th edition. Jefferson City, USA: John Wiley \& Sons, Inc.

Montgomery, D.C. \& Runger, G.C., 2014. Applied Statistics and Probability for Engineers, 6th edition. New Jersey, USA: John Wiley \& Sons, Inc.

Nagata, Y., 1991. Interval estimation for the process capability indices. Journal of Japanese Society for Quality Control, Vol. 21, pp. 109-114.

Nagata, Y. \& Nagahata, H., 1994. Approximation formulas for the confidence intervals of process capability indices. Okayama Economic Review, Vol. 25, No. 4, pp. 301-314.

NIST/SEMATECH., 2012. e-Handbook of Statistical Methods. Retrieved from http://www.itl.nist.gov/ div898/handbook.

Pearn, W.L. \& Chen, K.S., 1995. Estimating process capability indices for non-normal Pearsonian populations. Quality and Reliability Engineering International, Vol. 11, No. 5, pp. 389-391. https://doi.org/10.1002/qre.4680110510

Pearn, W.L. \& Kotz, S. 1994. Application of Clements' method for calculating second and third generation process capability indices for non-normal Pearsonian populations. Quality Engineering, Vol. 7, No. 1, pp. 139-145. https://doi.org/10.1080/08982119408918772

Peng, C., 2010. Parametric lower confidence limits of quantile-based process capability indices. Quality Technology \& Quantitative Management, Vol. 7, No. 3, pp. 199-214. https://doi.org/10.1080/16843703.2010.11673228

Perakis, M. \& Xekalaki, E., 2002. A process capability index that is based on the proportion of conforming. Journal of Statistical Computation and Simulation, Vol. 72, No. 9, pp. 707-718. https://doi.org/10.1080/00949650214270

Perakis, M. \& Xekalaki, E., 2005. A process capability index for discrete processes. Journal of Statistical Computation and Simulation, Vol. 75, No. 3, pp. 175-187. https://doi.org/10.1080/00949650410001687244

Polhemus, N.W. 2018. Process Capability Analysis: Estimating Quality. New York, USA: CRC press, Taylor \& Francis Group.

Quesenberry, C.P., 1991. SPC Q charts for a Binomial parameter p: short or long runs. Journal of Quality Technology, Vol. 23, No. 3, pp. 239-246. https://doi.org/10.1080/00224065.1991.11979329

Shi, L., Ma, H. and Lin, D-K. 2016. Process capability analysis via continuous ranked probability score. Quality and Reliability Engineering International, Vol. 34, No. 4, pp. 681-697. https://doi.org/10.1002/qre.1967

Shore, H., 1998. A new approach to analysing non-normal quality data with application to process capability analysis. International Journal of Production Research, Vol. 36, No. 7, pp. 1917-1933. https://doi.org/10.1080/002075498193039

Stoma P, Stoma M, Dudziak A, Caban J. 2019. Bootstrap analysis of the production processes capability assessment. Applied Sciences, Vol. 9, No. 24, pp. 1-15. https://doi.org/10.3390/app9245360

Vannman, K., 2006. Safety regions in process capability plots. Quality Technology \& Quantitative Management, Vol. 3, No. 2, pp. 227-246. https://doi.org/10.1080/16843703.2006.11673112

Wang, H., Yang, J. and Hao, S. 2016. Two inverse normalizing transformation methods for the process capability analysis of nonnormal process data. Computers \& Industrial Engineering, Vol. 102, pp. 88-98. https://doi.org/10.1016/j.cie.2016.10.014

Wu, C.W., Pearn, W.L. \& Kotz, S., 2009. An overview of theory and practice on process capability indices for quality assurance, International Journal of Production Economics, Vol. 117, No. 2, pp. 338-359. https://doi.org/10.1016/j.ijpe.2008.11.008

Yeh, F.B. \& Bhattacharya, S., 1998. A robust process capability index. Communications in Statistics - Simulation and Computation, Vol. 27, No. 2, pp. 565-589. https://doi.org/10.1080/03610919808813495

Yum, B.J. \& Kim, K.W. 2011. A bibliography of the literature on process capability indices: 2000-2009. Quality and Reliability Engineering International, Vol. 27, No. 3, pp. 251-268. https://doi.org/10.1002/qre.1115

Zhang, N.F., Stenback, G.A. \& Wardrop, D.M., 1990. Interval estimation of process capability index $C_{p l}$. Communication in Statistics- Theory and Method, Vol.19, pp. 4455-4470. https://doi.org/10.1080/03610929008830451 


\section{Biographical notes}

Dr. Surajit Pal is a Faculty Member in the Statistical Quality Control and Operations Research Unit of the Indian Statistical Institute, Chennai Centre, India. His fields of interest are quality engineering, process optimization, statistical quality control and multiple response optimization. He has published about forty five papers in different National and International journals.

Dr. Susanta Kumar Gauri is a Faculty Member in the Statistical Quality Control and Operations Research Unit of the Indian Statistical Institute, Kolkata, India. His fields of interest are quality engineering, process optimization, statistical quality control and multiple response optimization. He has published about sixty papers in different National and International journals. 